\title{
Improved Doppler Velocity Dealiasing for Radar Data Assimilation and Storm-Scale Vortex Detection
}

\author{
Qin Xu, ${ }^{1}$ Kang Nai, ${ }^{2}$ Shun Liu, ${ }^{3}$ Chris Karstens, ${ }^{1,2}$ Travis Smith, ${ }^{1,2}$ and Qingyun Zhao ${ }^{4}$ \\ ${ }^{1}$ National Severe Storms Laboratory, Norman, OK 73072, USA \\ ${ }^{2}$ Cooperative Institute for Mesoscale Meteorological Studies, University of Oklahoma, Norman, OK 73072, USA \\ ${ }^{3}$ National Centers of Environmental Prediction and I. M. Systems Group Inc., College Park, MD 20740, USA \\ ${ }^{4}$ Marine Meteorology Division, Naval Research Laboratory, Monterey, CA 93943, USA \\ Correspondence should be addressed to Qin Xu; qin.xu@noaa.gov
}

Received 28 May 2013; Revised 6 August 2013; Accepted 9 August 2013

Academic Editor: David J. Stensrud

Copyright ( 2013 Qin Xu et al. This is an open access article distributed under the Creative Commons Attribution License, which permits unrestricted use, distribution, and reproduction in any medium, provided the original work is properly cited.

\begin{abstract}
The Doppler velocity dealiasing technique based on alias-robust VAD and variational (AR-Var) analyses developed at the National Severe Storms Laboratory for radar data quality control and assimilation is further improved in its two-step procedures: the reference check in the first step and the continuity check in the second step. In the first step, the alias-robust variational analysis is modified adaptively and used in place of the alias-robust velocity-azimuth display (VAD) analysis for all scan modes (rather than solely the WSR-88D volume coverage pattern 31 with the Nyquist velocity $v_{N}$ reduced below $12 \mathrm{~m} \mathrm{~s}^{-1}$ and the TDWR Mod80 with $v_{N}$ reduced below $15 \mathrm{~m} \mathrm{~s}^{-1}$ ), so more raw data can pass the stringent threshold conditions used by the reference check in the first step. This improves the dealiased data coverage without false dealiasing to better satisfy the high data quality standard required by radar data assimilation. In the second step, new procedures are designed and added to the continuity check to increase the dealiased data coverage over storm-scale areas threatened by intense mesocyclones and their generated tornados. The performances of the improved dealiasing technique versus the existing techniques are exemplified by the results obtained for tornadic storms scanned by the operational KTLX radar in Oklahoma.
\end{abstract}

\section{Introduction}

Radar data quality control is critical for radar data assimilation, and dealiasing is an important and yet often very difficult part of radar data quality control. Currently, the local environment dealiasing algorithm (Eilts and Smith [1]) is used on WSR-88D radars (NEXRAD Network) for processing radar data for Doppler velocity data in real-time. When this algorithm was originally developed at the National Severe Storms Laboratory (NSSL), the primary goal was for visual and certain quantitative applications, such as automated mesocyclone detections (Stumpf et al. [2] and Smith and Elmore [3]), with considerable tolerance for bad or poor quality data to retain as much as possible the original data coverage. Therefore, the processed data often do not satisfy the high-quality standard required by data assimilation at the National Centers of Environmental Prediction (NCEP). This problem is common for most early developed dealiasing techniques, especially for those designed primarily for applications not involving data assimilation including the currently developed and tested techniques (such as the twodimensional dealiasing of Jing and Wiener [4]) for broad applications at the NWS Radar Operations Center (Burgess and Crum [5] and Witt et al. [6]). Striving to meet the need of radar data assimilation, continued efforts have been made at NSSL in collaboration with other research institutes to develop robust dealiasing techniques (Gong et al. [7], Gao et al. [8], Zhang and Wang [9], Xu et al. [10], and $\mathrm{Xu}$ and Nai [11]). In particular, the dealiasing technique of Xu et al. [10] has been tested extensively with real-time observations from operational WSR-88D radars under various weather conditions. From these tests, the technique was found to be capable of correcting alias errors without false dealiasing in most cases, but it occasionally failed to correct or flag severely aliased radial velocities around strongly sheared and often tilted inversion layers in severe winter ice storms scanned by the operational WSR-88D radars using volume coverage 
pattern 31 (VCP31) with $v_{N}<12 \mathrm{~m} \mathrm{~s}^{-1}$, where $v_{N}$ denotes the Nyquist velocity. The occasional failures were caused by the lack of the required accuracy (within $\pm 7 v_{N} / 4$ of the true value at every observation point) of the reference radial-velocity field produced by the alias-robust velocity-azimuth display (AR-VAD) analysis (Xu et al. [12]). To solve this problem (caused mainly by reduced $v_{N}$ ), an alias-robust variational (AR-Var) analysis was developed (Xu and Nai [13]) and used in place of the AR-VAD analysis for the reference check to upgrade the VAD-based dealiasing (Xu et al. [10]) adaptively for VCP31 into an AR-Var-based dealiasing (Xu and Nai [11]). As the above dealiasing techniques were developed for data assimilation applications at NCEP (Liu et al. [14]), various stringent threshold conditions must be used to ensure the dealiased data to be completely free of false dealiasing. As these stringent conditions tend to discard many data that cannot pass the thresholds even after dealiasing, the dealiased data often have less or even much less coverage than the raw data, especially in isolated data areas far away from the radar or in localized areas of strong and complex winds such as those around mesocyclones. It is thus necessary to further improve these dealiasing techniques to increase their dealiased data coverage but remain to be completely free of false dealiasing, especially over storm-scale areas threatened by intense mesocyclones and their generated tornados. The above existing dealiasing techniques for data assimilation are reviewed in Section 2. Improvements made to the existing dealiasing techniques are presented in Section 3. Conclusions follow in Section 4.

\section{Review of VAD-Based and AR-Var-Based Dealiasing Techniques for Data Assimilation}

The VAD-based dealiasing technique (Xu et al. [10]) developed at NSSL for radar wind data quality control was delivered to NCEP for radar data assimilation tests and applications (Liu et al. [14]). This dealiasing performs two steps: (i) the reference check with the reference radial-velocity field produced by the AR-VAD analysis (Xu et al. [12]) to detect and correct alias errors in the all-flagged raw data and (ii) the continuity check with an enhanced use of available seed data (i.e., dealiased or deflagged data that have passed the reference check and thus have been accepted as good data) in a properly enlarged block area near each remaining flagged data point being checked. The technique has been tested with raw radial velocities scanned from operational WSR-88D radars under various weather conditions. In most cases, the algorithm can correct aliased velocities without false dealiasing. However, like the classic VAD analysis, the AR-VAD analysis is inherently limited by its uniform-wind assumption, so the resultant reference radial velocities often do not have the desired flexibility and variability to allow most raw data to pass the stringent threshold conditions, especially when the Nyquist velocity $v_{N}$ is reduced, and thus, the threshold ranges are further narrowed. Because of this, the VAD-based dealiasing occasionally fails to detect and correct severely aliased velocities around strongly sheared and tilted inversion layers in severe winter ice storms observed by operational WSR-88D radars using VCP31 with $v_{N}<$ $12 \mathrm{~m} \mathrm{~s}^{-1}$. To solve this problem, the AR-Var analysis (Xu et al. [15]) was refined into a two-step analysis (Xu and Nai [13]) and used in place of the AR-VAD analysis for the reference check. In the first step of the refined ARVar analysis, the original AR-VAD analysis was modified to fit the raw aliased radial-velocity observations in the $\pm 90^{\circ}$ vicinity of each of the two paired zero radial-velocity points (about $180^{\circ}$ apart) on a selected range circle. This modification resembles the sectorized uniform-wind technique applied with two $180^{\circ}$ sectors. The two analyzed radial-velocity fields were then combined into a single radial-velocity field not rigidly constrained by the VAD uniform-wind assumption. In the second step, the combined radial-velocity field was used as the first guess for the refined AR-Var analysis. This upgraded the AR-VAD-based dealiasing into the AR-Varbased dealiasing (Xu and Nai [11]) adaptively for winter ice storms scanned by WSR-88D radars using VCP31 (at $0.5^{\circ}, 1.5^{\circ}$, $2.5^{\circ}, 3.5^{\circ}$, and $4.5^{\circ}$ tilts) as well as for storms scanned by the FAA Terminal Doppler Weather Radar (TDWR) radars using Mod80 (with $v_{N}<15 \mathrm{~m} \mathrm{~s}^{-1}$ at $0.5^{\circ}, 1.0^{\circ}, 2.5^{\circ}, 5.1^{\circ}, 7.7^{\circ}, 11.3^{\circ}$, $15.3^{\circ}, 20.7^{\circ}$, and $28.2^{\circ}$ tilts).

The AR-Var-based dealiasing has been tested successfully with many winter ice storms scanned by operational WSR$88 \mathrm{D}$ radars using VCP31 with $v_{N}<12 \mathrm{~m} \mathrm{~s}^{-1}$ and many storms scanned by the FAA OKC airport TDWR radar using Mod80 with $v_{N}<15 \mathrm{~m} \mathrm{~s}^{-1}$. The effectiveness of this dealiasing technique is shown by the example in Figure 1 (in addition to the example in Figure 2 of $\mathrm{Xu}$ and Nai [11]). As shown in Figure 1(a)(or Figure 1(b)), the raw radial velocities were severely aliased in most areas outside the 25 (or 10) $\mathrm{km}$ radial range, and the aliased velocities were even folded twice in areas to the southwest and northeast of the radar outside the 150 (or 60 ) $\mathrm{km}$ radial range on the $1.5^{\circ}$ (or $4.5^{\circ}$ ) tilt. As shown by the dealiased radial-velocity field in Figure 1(c) (or Figure $1(\mathrm{~d})$ ), the wind field is dominated by a strong northeasterly flow below the vertical level of $z \approx 0.7 \mathrm{~km}$ (that is, within the radial range of $r \approx 25$ (or 15) $\mathrm{km}$ on $1.5^{\circ}$ (or $4.5^{\circ}$ ) tilt) and then veers sharply to a strong southwesterly flow above $z \approx 0.8 \mathrm{~km}$. This sharp change in wind direction is a typical feature often observed during winter ice storms, and this feature is well captured by the two-step AR-Var analysis but not retrieved by the AR-VAD analysis due to the reduced $v_{N}\left(=11.51 \mathrm{~m} \mathrm{~s}^{-1}\right)$ and the increased discrepancy between the VAD-analyzed uniform wind and the true wind, especially around the vertical-shear layer that is not perfectly flat.

The AR-Var-based dealiasing has been incorporated into the radar data quality control package (Liu et al. [14]) at the National Centers for Environmental Prediction (NCEP) for operational tests. For radar data assimilation applications at NCEP, the method is required to be absolutely free of false dealiasing. This has inevitably sacrificed the data coverage to certain but different degrees for AR-Var-based dealiasing and AR-VAD-based dealiasing. The sacrificed (flagged) data coverage is usually minor and marginal for the AR-Var-based dealiasing applied adaptively to winter ice storms since the raw velocities scanned from ice storms using VCP31 usually 


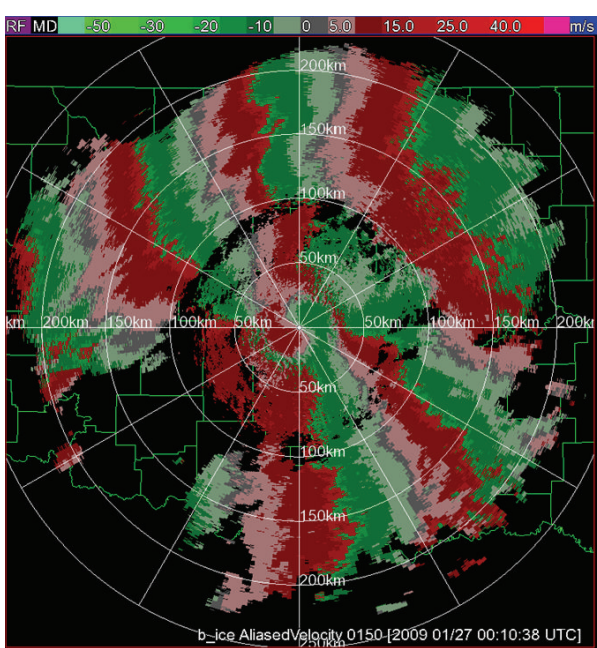

(a)

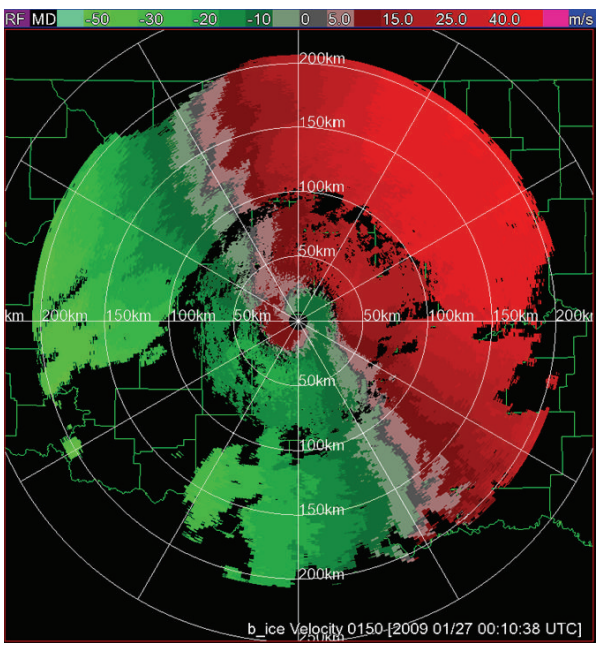

(c)

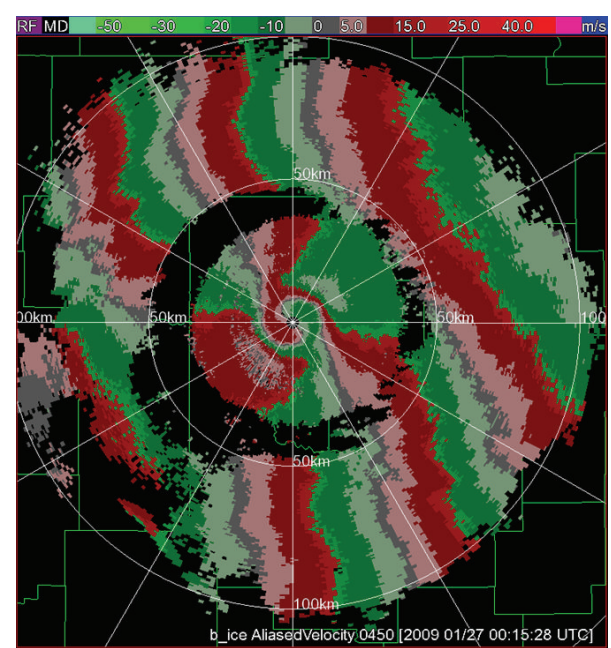

(b)

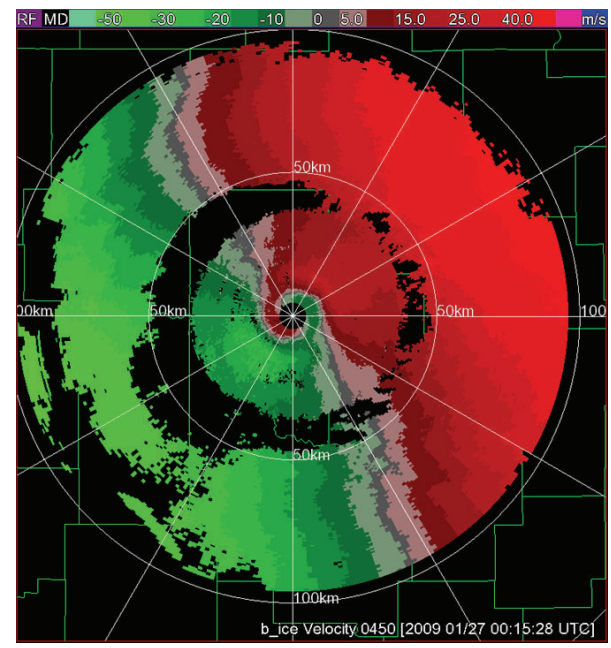

(d)

FIgURE 1: Raw velocity image scanned by the KTLX radar with $v_{N}=11.51 \mathrm{~m} \mathrm{~s}^{-1}$ (a) on $1.5^{\circ}$ tilt at 00:10:38 UTC and (b) on $4.5^{\circ}$ tilt at $00: 15: 28$ UTC for the ice storm on January 27, 2009. Dealiased velocity image on (c) $1.5^{\circ}$ and (d) $4.5^{\circ}$ tilts by the AR-Var-based dealiasing.

have rather continuous and complete coverage on each tilt, and so do the dealiased data (as shown in Figure 1). However, for the VAD-based dealiasing still used for other VCPs, the reference check in the first main step has to be limited within the cut-off radial range $\left(30 \mathrm{~km}\right.$ for $0.5^{\circ}$ tilt and $80 \mathrm{~km}$ for all other higher tilts; see Section $2 \mathrm{~b}$ of Xu et al. [10]) to avoid false dealiasing, so isolated data areas away (by 40 gates and 5 beams or more) from the seed data areas produced by the reference check will remain flagged as they cannot be reached by the continuity check in the second step of the VAD-based dealiasing (see Section $2 \mathrm{c}$ of Xu et al. [10]). This limitation can become serious for a spring or summer convective storm when the raw velocities scanned from the storm using VCP11, VCP211, VCP12, or VCP121 (with $v_{N}>20 \mathrm{~m} \mathrm{~s}^{-1}$ at 14 tilts from $0.5^{\circ}$ to $19.5^{\circ}$ ) are scattered sparsely in large isolated areas far away from the radar. To reduce this limitation, further improvements are made to both the reference check in the first step and the continuity check in the second step. These new improvements are described in Section 3.

\section{New Improvements and Results}

To increase the coverage of seed data produced by the reference check, the AR-Var-based dealiasing is extended and used in place of the VAD-based dealiasing for all the remaining VCPs (other than VCP31 for WSR-88D and Mod80 for TDWR) with two new adaptations (for $v_{N}>18 \mathrm{~m} \mathrm{~s}^{-1}$ only): (i) The first-guess background radial-velocity field (used to start the descent algorithm in the second step of the refined AR-Var analysis) is taken directly from the AR-VAD analysis without modification in the first step of the refined AR-Var analysis, because the modified AR-VAD analysis is necessary only for $v_{N}<18 \mathrm{~m} \mathrm{~s}^{-1}$ but it requires sufficient raw-data coverage on each selected range circle as explained in Section 2. (ii) The cut-off radial range limitation (see Section $2 \mathrm{~b}$ of $\mathrm{Xu}$ et al. [10]) is removed for the reference check, because the refined AR-Var analysis is not rigidly constrained by the VAD uniform-wind assumption and thus can fit the data much better than the AR-VAD analysis at 


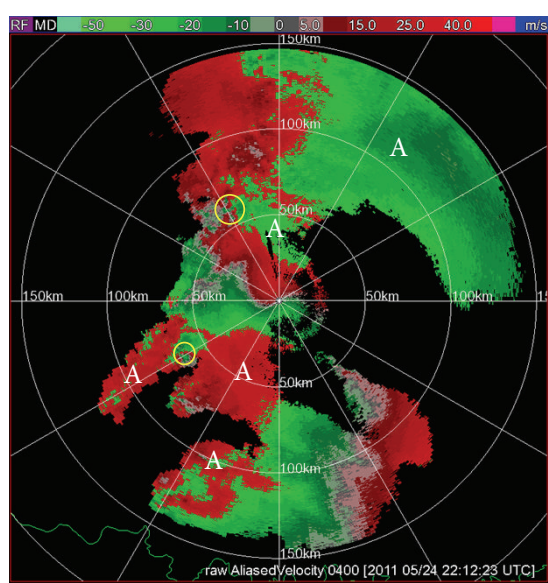

(a)

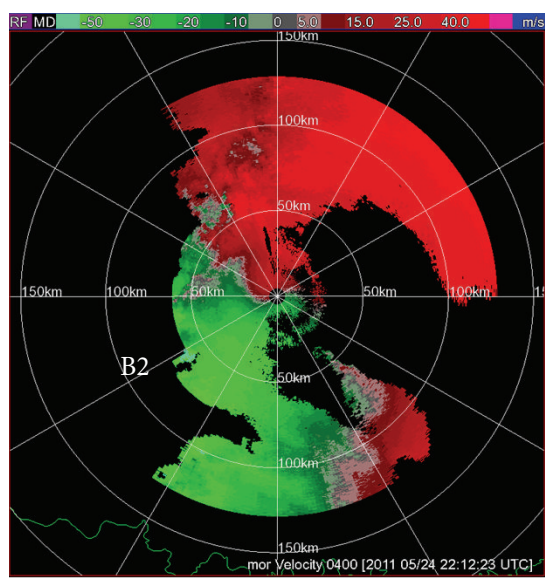

(d)

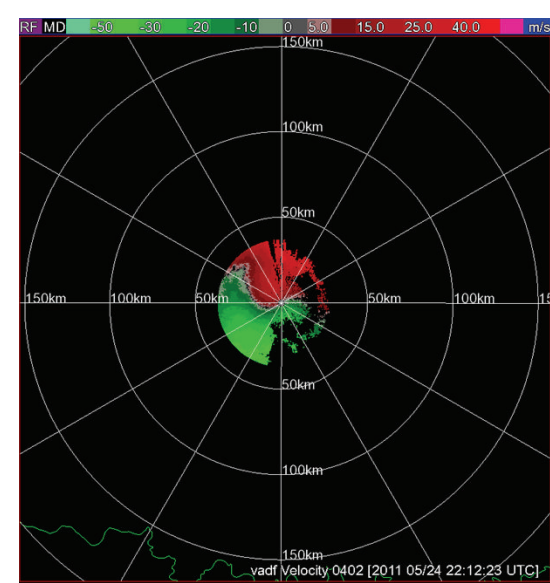

(b)

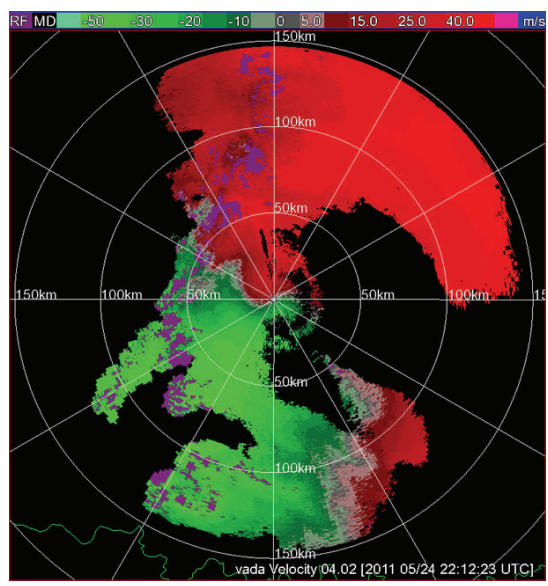

(e)

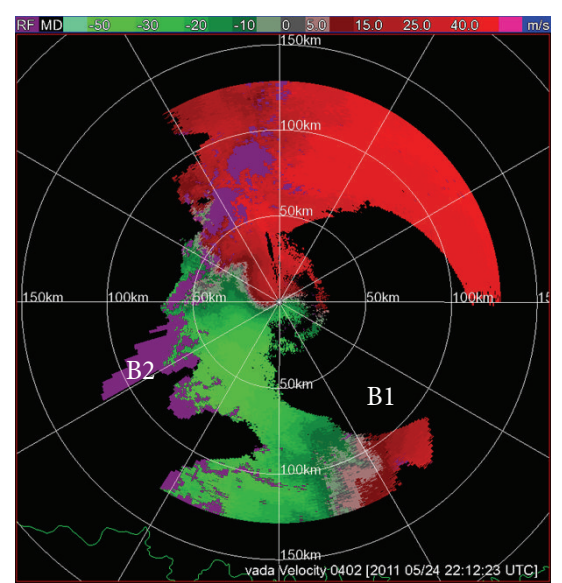

(c)

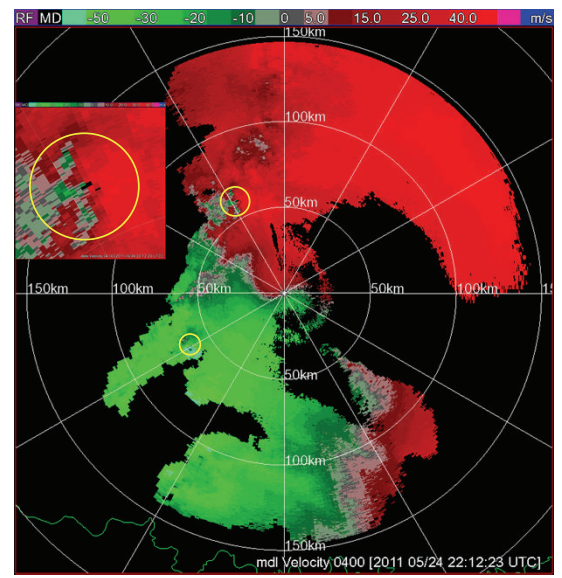

(f)

FIgURE 2: (a) Raw radial-velocity image scanned by the KTLX radar using VCP12 with $v_{N}=26.1 \mathrm{~m} \mathrm{~s}^{-1}$ at $4.0^{\circ}$ tilt for the Oklahoma tornadic storm system at 22:12:23 UTC on May 24, 2011. (b) Dealiased radial-velocity (seed data) image produced by the improved reference check alone. (c) Dealiased radial-velocity image (with the special data points plotted in purple) produced by the first one-way forward procedure in the continuity check after the improved reference check. (d) Final dealiased radial-velocity image produced by the new extended AR-Varbased method. (e) As in panel (c) but the first guess is from the RAP-predicted wind field for the refined AR-Var analysis. (f) As in panel (d) but the first guess is from the RAP-predicted wind field for the refined AR-Var analysis. The white letters "A" in panel (a) mark the main aliased-velocity areas. The white letters B1 and B2 in panel (c) mark the two flagged (in black) peninsula areas. The white letter B2 in panel (d) marks the remained flagged peninsula area. The large (or small) yellow circle marks a mature (or emerging) tornadic mesocyclone in panels (a) and (f), and a magnified frame is inserted to show the mature tornadic mesocyclone in panel (f). The green contour on the bottom of each panel shows the boundary between Oklahoma and Texas states. The color scale for the radial velocity is plotted on the top of each panel.

far radial ranges (with an increased analysis accuracy as exemplified in Figures 1 and 2 of $\mathrm{Xu}$ and $\mathrm{Nai}$ [13]). The above two adaptations can improve the seed data coverage, but the improved seed data coverage is still confined by the often limited radial range of the first-guess background radial-velocity field produced by the AR-VAD analysis. This is shown by the example in Figure 2(b), where the seed data produced from the raw data in Figure 2(a) by the reference check with the above two adaptations are still confined within the $36 \mathrm{~km}$ radial range, because the AR-VAD analysis fails to produce the first-guess background radial-velocity field for the AR-Var analysis for $r>36 \mathrm{~km}\left(z>2.6 \mathrm{~km}\right.$ on $4.0^{\circ}$ tilt). As listed in column 3 of Table 1, these seed data cover $28.04 \%$ of the raw data, which is better than the seed data, overage (26.36\%) produced by the reference check in the ARVAD-based dealiasing (not shown). Nevertheless, after the continuity check is performed (one-way forward going away from the radar) in the second main step, the new extended AR-Var-based dealiasing can cover most connected raw-data areas without any false dealiasing as shown in Figure 2(c), and this coverage $(76.07 \%$ as listed in column 5 of Table 1 ) is higher than that $(74.56 \%)$ of the AR-VAD-based dealiasing. As we can see in Figure 2(c), there are still flagged (in black or purple) data holes (especially around the two tornadic mesocyclones marked by the large and small yellow circles in Figure 2(a)) and large amounts of flagged data in the two peninsula areas (marked by the white letters B1 and B2 in Figure 2(b)) and beyond the $130 \mathrm{~km}$ radial range. 
To recover the flagged data holes (especially around tornadic mesocyclones), the original one-way procedure (going forward away from the radar) of the continuity check (see Section $2 c$ of Xu et al. [10]) is upgraded into a two-way procedure (going forward and backward twice on each tilt). In addition, the block-to-point continuity check in Section $2 c(1)$ of $\mathrm{Xu}$ et al. [10] and the three-directional continuity check in Section $2 \mathrm{c}(2)$ of $\mathrm{Xu}$ et al. [10] are modified and augmented with new step as described below.

(i) The threshold value for the modified block-to-point continuity check is tightened from $v_{N} / 2$ to $v_{N} / 3$ in (6) of Xu et al. [10], and flagged data that fail to pass this tightened threshold condition are marked as special data points (to be used and treated in later steps). The Nyquist folding number correction and related check in (7) of Xu et al. [10] are not performed here but delayed to the new fifth step described below. This can avoid possible false dealiasing in the vicinity of a mesocyclone.

(ii) If at least 40 seed data cannot be found in the $40 \Delta r \times$ $11^{\circ}$ box, and thus, the modified block-to-point continuity check in (i) cannot perform, then the three-directional continuity check is modified and performed with $v_{r}^{o 2}$ in (8)(10) of Xu et al. [10] replaced by

$$
v_{r}^{o N}=v_{r}^{o}+2 N_{v_{N}}
$$

where $v_{r}^{o}$ is the raw radial-velocity observation, $N=$ $\operatorname{Int}\left[\left(v_{r}^{\text {seed }}-v_{r}^{o}\right) /\left(2 v_{N}\right)\right]$ is the Nyquist folding number estimated for $v_{r}^{o}$ from $v_{r}^{\text {seed }}$, Int $[()]$ represents the nearest integer of (), and $v_{r}^{\text {seed }}$ is given by the seed datum (i.e., $v_{r}^{\text {seed1 }}$ in (8), $v_{r}^{\text {seed2 }}$ in (9), or $v_{r}^{\text {seed3 }}$ in (10) of Xu et al. [10]). The flowchart of the above steps (i)-(ii) is shown in Figure 3(a).

(iii) A new third step is added to recheck each seed datum. The rechecking procedure goes forward (away from the radar) along each beam through the entire tilt (clockwise). The seed datum being checked will degrade to a special datum if the following two conditions are both met. (a) There is one or more special data points within 10 gates (along the same beam from the seed datum) in the direction opposite to the searching direction. (b) There is one or more special data point within 15 gates in the searching direction or there are more than 5 flagged data points within 15 gates and more than 20 flagged data points within 40 gates in the searching direction. This third step ensures each accepted seed datum to be not two-sided by special data along the current beam (where the special datum is located) and not neighbored by too many flagged data in the searching direction.

(iv) A new fourth step is added to perform a line-topoint continuity check at each special datum point. The procedure goes forward along each beam through the entire tilt (clockwise). The special datum being checked or its correction $v_{r}^{o N}$ will be upgraded to a seed datum if the following two conditions are both satisfied. (a) $v_{r}^{o N}$ is within $\pm v_{N} / 2$ of the averaged value of the seed data (if any) available from the three data points that are on the current beam and its two neighboring beams along either of the two adjacent range circles (to the current range circle where the special datum is located). (b) $v_{r}^{o N}$ is within $\pm v_{N} / 2$ of the averaged value of the seed data (if any) available from the three data points that are on the current range circle and its two neighboring range circles along either of the two adjacent beams (to the current beam). Here, $v_{r}^{o N}$ is computed by (1) with $N$ estimated for $v_{r}^{o}$ from $v_{r}^{\text {seed }}, v_{r}^{o}$ is the special datum being checked, and $v_{r}^{\text {seed }}$ is given by the averaged value of the seed data.

(v) A new fifth step is added to perform a point-to-point continuity check at each special datum point. The procedure goes in the same way as in the above step (iv). The special datum being checked or its correction $v_{r}^{o N}$ will upgrade to a seed datum if the following two conditions are both satisfied. (a) $v_{r}^{o N}$ is within $\pm v_{N} / 2$ of the nearest seed datum that is within \pm 5 range gates from the special datum point along the same beam. (b) $v_{r}^{o N}$ is within $\pm v_{N} / 2$ of the nearest seed datum that is within \pm 2 beam positions from the special datum point along the same range circle. Here, $v_{r}^{o N}$ is computed by (1) with $N$ estimated for $v_{r}^{o}$ from $v_{r}^{\text {seed }}, v_{r}^{o}$ is the special datum being checked, and $v_{r}^{\text {seed }}$ is given by the seed datum. The above steps (iv)-(v) are performed repetitively through the entire tilt for three times. This completes the first one-way forward procedure, as shown by the flowchart in Figure 3(b).

(vi) After the above first one-way forward procedure, the procedure is restarted with the five steps (i)-(vi) performed one way backward (toward the radar). After this, the above two-way forward-backward procedure is repeated in the same way as described above except that the line-to-point continuity check and point-to-point continuity check in (iv)(v) are now applied only to those special data that satisfy the four cyclonic-rotation conditions (listed in the appendix) in an $11 \times 41$ window (11 beams and 41 range gates) centered at the special datum point being checked.

The effectiveness of the above modifications (i)-(vi) is exemplified by the result in Figure 2(d), where the data holes that are previously identified by the first one-way forward procedure as special data points (plotted in purple in Figure 2(c)) are all recovered around the mature tornadic mesocyclone and the previously flagged data in the first peninsula area (marked by the white letters B1 in Figure 2(c)) are also recovered, although the flagged data and special data in the second peninsula area (marked by the white letters B2 in Figure 2(d)) and the flagged data beyond the $130 \mathrm{~km}$ radial range are not recovered yet. The final dealiased data (including the deflagged nonaliased data) cover $83.66 \%$ of the raw data as listed in column 6 of Table 1 . The CPU time required by this new extended AR-Var-based dealiasing is $99 \mathrm{~s}$ for processing the entire volume of radial-velocity data for the case in Figure 2, which is nearly 2.5 times of that (40 s) required by the AR-VAD-based dealiasing but is still fast enough for real-time applications. The increased CPU time is caused by the AR-Var analysis that can be but is not yet parallelized in the current code and thus is applied sequentially to each selected range circle on each tilt. The increased part of the CPU time can be reduced by about 10 (or even $10^{2}$ ) times if the AR-Var analysis is parallelized for all different tilts (or all selected range circles).

In addition to the above improvements, a modelpredicted wind field can be interpolated onto each selected 
TABLE 1: Numbers of raw data (column 2), seed data (column 3), one-way special data (column 4), one-way dealiased data (column 5), final dealiased data (column 6), and falsely dealiased data (column 7) are produced by the three methods (column 1) for the case in Figure 2. The percentage with respect to the total number of raw data is also listed below the number in each type of data in the same box. "Seed data" are dealiased data produced by the reference check alone. "One-way special data" are special data identified by the first one-way forward procedure (see the flowchart in Figure 3(b)) in the continuity check. "One-way dealiased" data are dealiased data produced by the first one-way forward procedure in the continuity check. All "dealiased" data include de-flagged nonaliased data.

\begin{tabular}{|c|c|c|c|c|c|c|}
\hline Method & Raw data & Seed data & One-way special data & One-way dealiased & Final dealiased & Falsely dealiased \\
\hline AR-VAD-based & 120971 & $\begin{array}{c}31882 \\
26.36 \%\end{array}$ & N/A & $\begin{array}{c}90191 \\
74.56 \%\end{array}$ & N/A & 0 \\
\hline New AR-Var-based & 120971 & $\begin{array}{c}33922 \\
28.04 \%\end{array}$ & $\begin{array}{l}10593 \\
8.75 \%\end{array}$ & $\begin{array}{c}92028 \\
76.07 \%\end{array}$ & $\begin{array}{l}101200 \\
83.66 \%\end{array}$ & 0 \\
\hline Model + AR-Var-based & 120971 & $\begin{array}{l}104436 \\
86.33 \% \\
\end{array}$ & $\begin{array}{c}6579 \\
5.44 \% \\
\end{array}$ & $\begin{array}{c}113712 \\
94.00 \%\end{array}$ & $\begin{array}{l}119964 \\
99.17 \%\end{array}$ & 0 \\
\hline
\end{tabular}

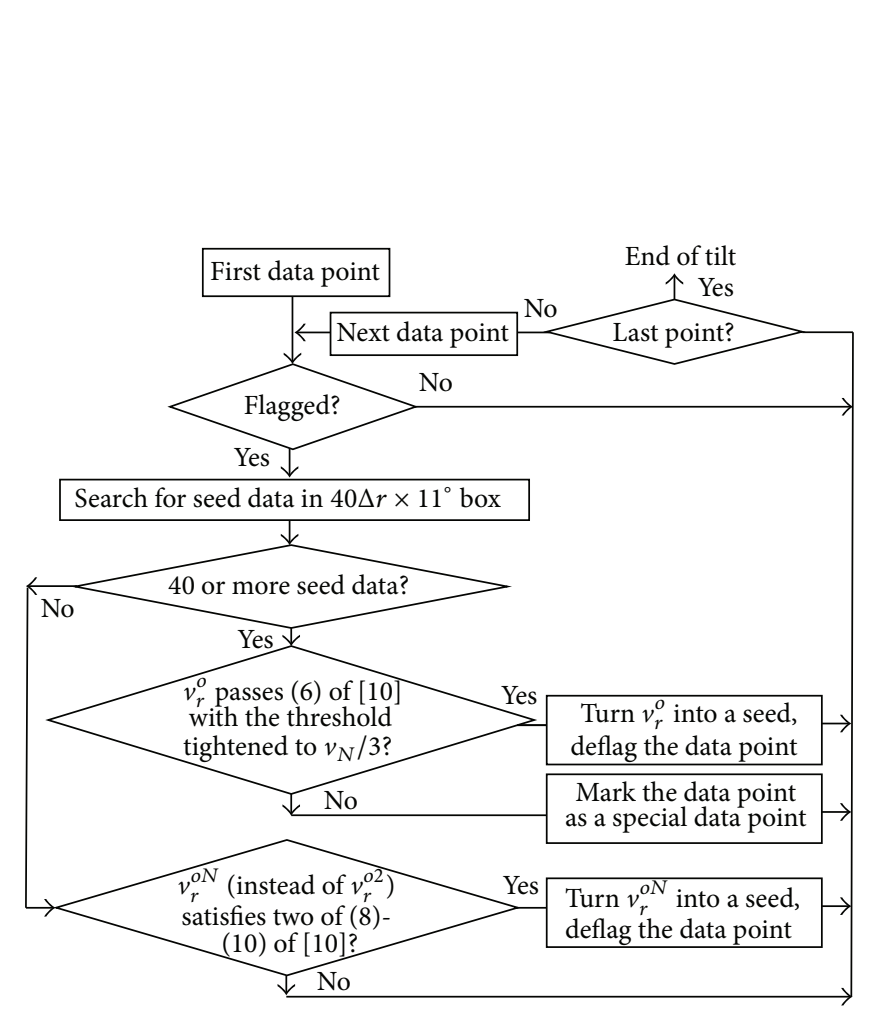

(a)

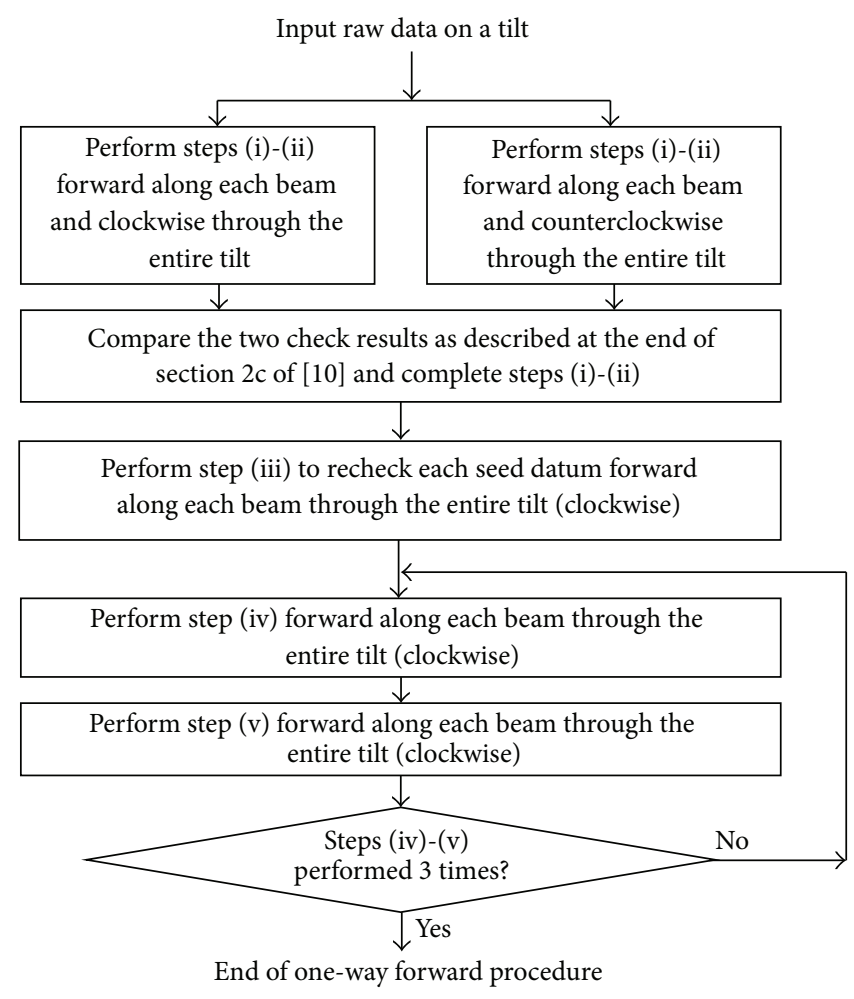

(b)

FIGURE 3: (a) Flowchart for the modified block-to-point continuity check and modified three-directional continuity check in steps (i)-(ii). (b) Flowchart for the first one-way forward procedure from step (i) to step (v) in the continuity check.

range circle and used as the first guess for the refined ARVar analysis to cover the isolated data areas far away from the radar if the predicted field can fit the data closely and allow most of the data to pass the threshold in (14) of Xu and Nai [13] for the refined AR-Var analysis. This approach was recently developed and tested with the wind fields predicted hourly by the NCEP operational Rapid Refresh (RAP) model. The preliminary results are very encouraging. The effectiveness of this approach, called model+AR-Varbased dealiasing, is exemplified by the improved results in Figures 2(e) and 2(f). In this case, the seed data coverage is increased from $28.04 \%$ to $86.33 \%$ (see column 3 of Table 1 ), the special data coverage is reduced from $8.75 \%$ to $5.44 \%$ (see column 4 of Table 1), the dealiased data coverage is increased from $76.07 \%$ to $94.00 \%$ (see column 5 of Table 1 ) after the first one-way forward procedure, and the final dealiased data coverage is increased from $83.66 \%$ to $99.17 \%$ (see column 6 of Table 1 ). The CPU time required by this model+AR-Var-based dealiasing is $271 \mathrm{~s}$ for processing the entire volume of radial-velocity data for the case in Figure 2, which is 6.8 times of that $(40 \mathrm{~s})$ required by the AR-VADbased dealiasing and is marginal for real-time applications. Again, the increased part of the CPU time can be reduced by about 10 (or even $10^{2}$ ) times if the AR-Var analysis used in this model+AR-Var-based dealiasing is parallelized for all different tilts (or all selected range circles). 


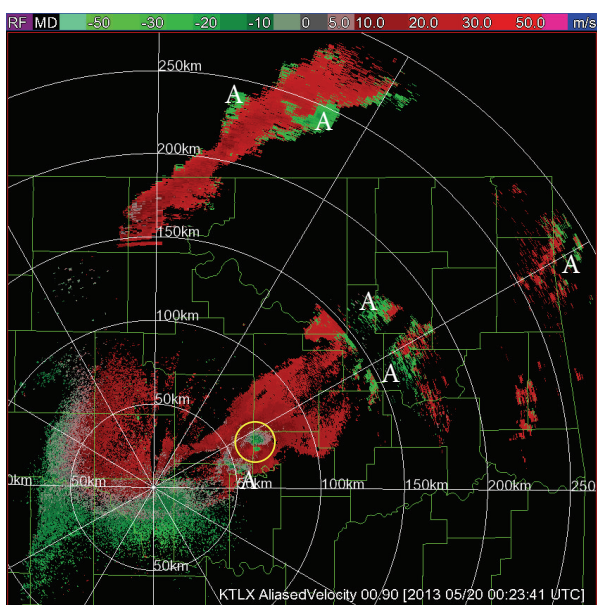

(a)

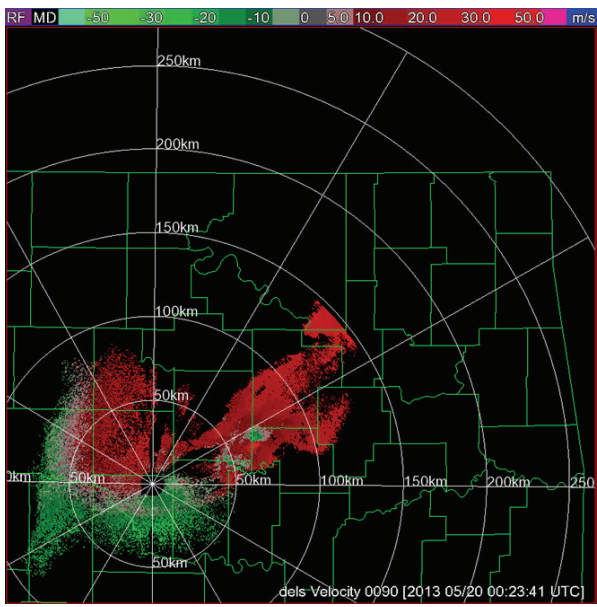

(c)

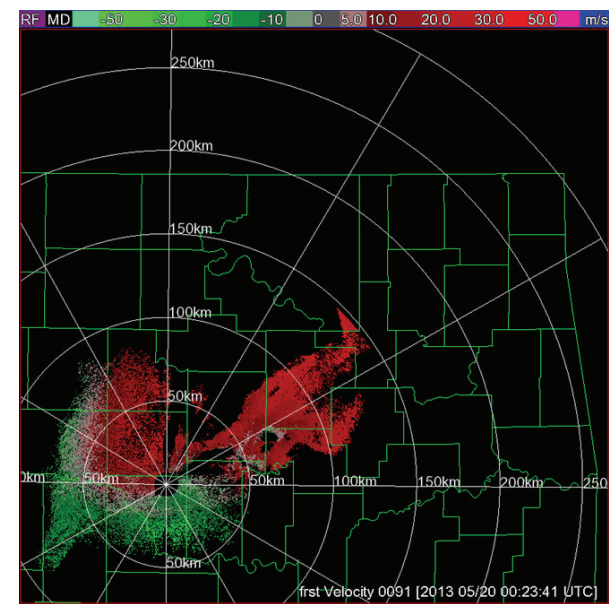

(b)

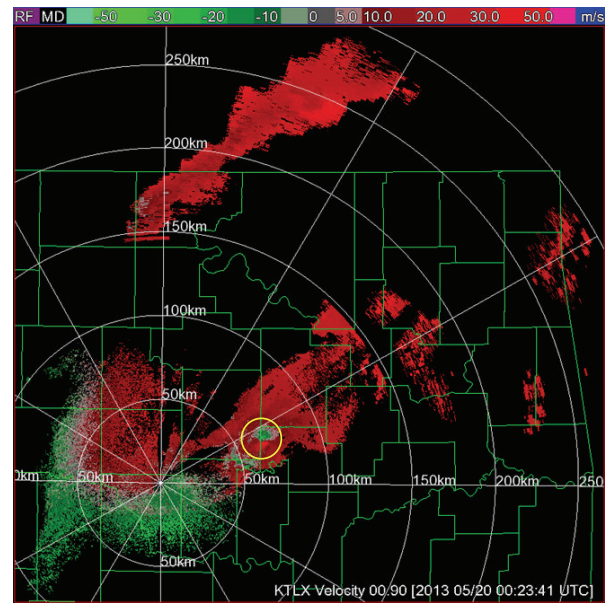

(d)

FIGURE 4: Raw radial-velocity image scanned by the KTLX radar using VCP12 with $v_{N}=26.1 \mathrm{~m} \mathrm{~s}^{-1}$ at $0.9^{\circ}$ tilt for the Shawnee, Oklahoma tornadic storm at 00:23:41 UTC on May 20, 2013 (local time 7:23:41 pm, on May 19, 2013). (b) Dealiased radial-velocity image produced by the first one-way forward procedure in the continuity check. (c) Final dealiased radial-velocity image produced by the new extended AR-Varbased method. (d) as in panel (d) but the first guess is from the RAP-predicted wind field for the refined AR-Var analysis. The white letters "A" in panel (a) mark the main aliased-velocity areas. The yellow circle in panels (a) and (d) encircles the mesocyclone and its produced EF4 tornado that struck Shawnee, Oklahoma, in the evening (local time around 6:45 pm) on May 19, 2013.

The improved dealiasing techniques presented in this paper have been running continuously with real-time data from five operational WSR-88D radars (KTLX, KSRX, KINX, KVNX, and KFDR) and one TDWR radar (TOKC). Their improved performances have been monitored and verified under various weather conditions, especially during severe storms. Examples of their real-time results are shown in Figures 4 and 5 for two recent tornadic storms scanned by the KTLX radar. The example in Figure 4 is the tornadic storm that produced a strong mesocyclone (marked by the yellow circle in Figure 4(d)) and an EF4 tornado that tracked from Norman to Shawnee, Oklahoma, in the evening (local time between 6:00 pm and 7:00 pm) on May 19, 2013. The example in Figure 5 is the tornadic storm that produced a strong mesocyclone (marked by the yellow circle in Figure 5(d)) and an EF5 tornado that struck the cities of Newcastle and Moore,
Oklahoma, in the afternoon (local time between 2:45 pm and 3:35 pm) on May 20, 2013.

\section{Conclusions}

In this paper, further improvements are made in radar velocity dealiasing atop of the recently published VADbased dealiasing (Xu et al. [10]) and AR-Var-based dealiasing (Xu and Nai [11]). The improved dealiasing techniques have been running continuously with real-time data from six operational radars (KTLX, KSRX, KINX, KVNX, KFDR, and TOKC). According to the real-time results so far monitored (as sampled in Figures 2, 4-6), the techniques are quite effective in improving the dealiased data coverage without false dealiasing. The improvement achieved by using the predicted wind field from the operational RAP model as the first 


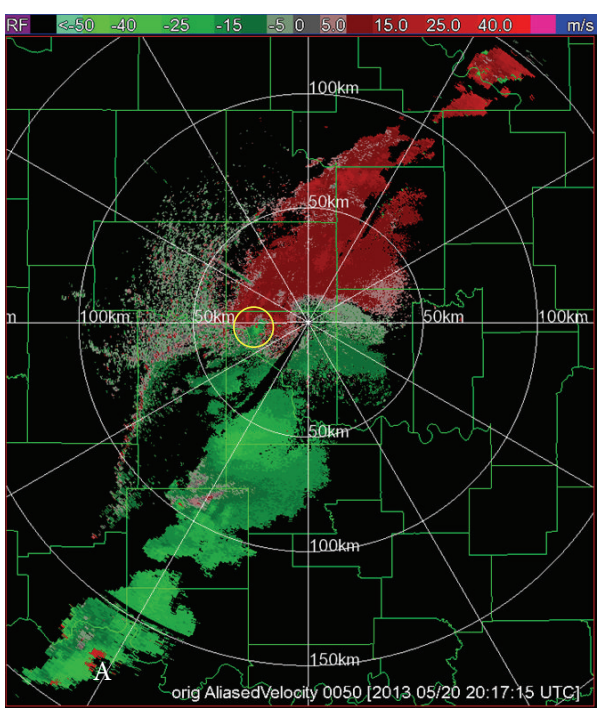

(a)

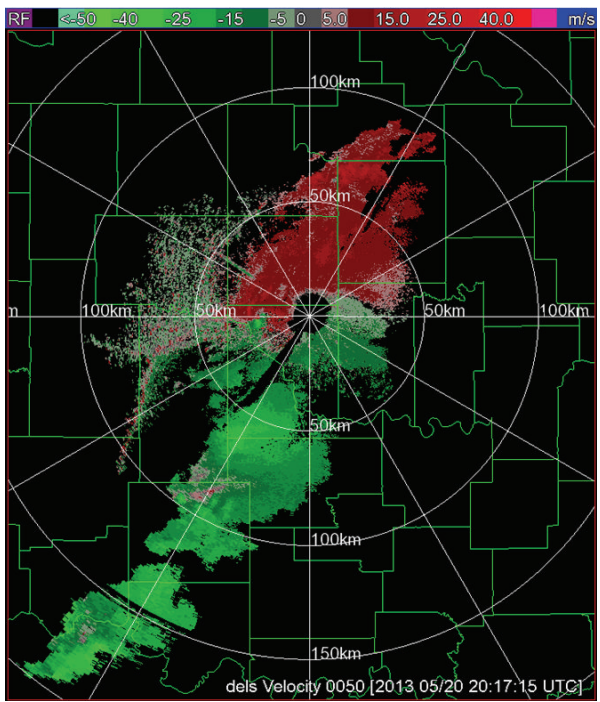

(c)

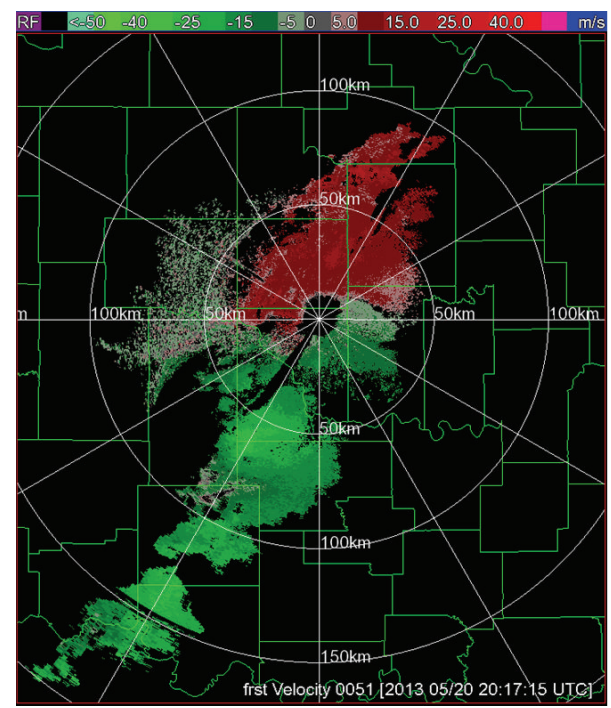

(b)

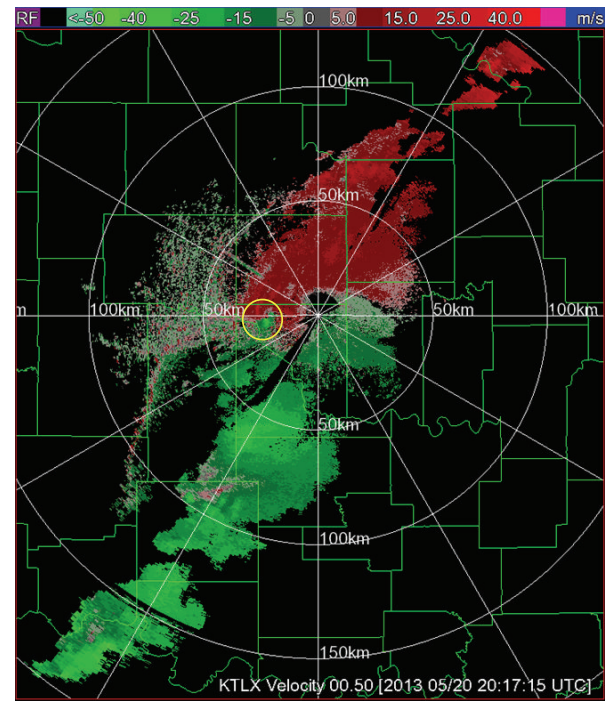

(d)

Figure 5: As in Figure 4 but for the Moore, Oklahoma, tornadic storm scanned by the KTLX radar using VCP12 with $v_{N}=26.1 \mathrm{~m} \mathrm{~s}^{-1}$ on $0.5^{\circ}$ tilt at 20:17:15 UTC on May 20, 2013 (local time 03:17:15 pm on May 20, 2013). The yellow circle in panels (a) and (d) encircles the mesocyclone and its produced EF5 tornado that struck Moore, Oklahoma, in the afternoon (local time around 3:30 pm) on May 20, 2013.

guess for the refined AR-Var analysis (Xu and Nai [13]) to produce a radial-velocity reference field over broad radial ranges for the reference check in the first step is especially encouraging. However, according to our additional tests (not shown in this paper), directly using the RAP-predicted wind field for the reference check without the refined AR-Var analysis can occasionally cause false dealiasing. In particular, directly using the RAP-predicted wind field in the reference check can work well (with no false dealiasing) for almost all the 222 volumes of raw radial-velocity data collected by the KTLX radar for the three cases considered in this paper, but it causes false dealiasing on 10 tilts in two volumes at 23:14:34 UTC on May 19, 2013. Although the false dealiasing rate is extremely low $(0.9 \%$ in number of volumes and $0.32 \%$ in number of tilts) for these three cases due to the stringent threshold condition used for the reference check (see (4)(5) of $\mathrm{Xu}$ et al. [10]), the false dealiasing area (less than 40 seed data points or 70 final dealiased data points on any of the 10 tilts) is within the mesocyclone (not shown). Thus, the refined AR-Var analysis is still necessary for the reference check to be free of false dealiasing when a model-predicted wind field is used. The current refined AR-Var analysis uses stringent threshold to filter radial-velocity data (see (14) of Xu and Nai [13]) and requires at least 20 valid radial-velocity data on each selected range circle, so it tends to reject scattered data in small isolated areas far away from the radar. This is a minor limitation for the improved reference check in the first step. It is possible to reduce this limitation by performing the AR-Var analysis not over the entire range circle but locally and adaptively around each isolated data area. The improved 


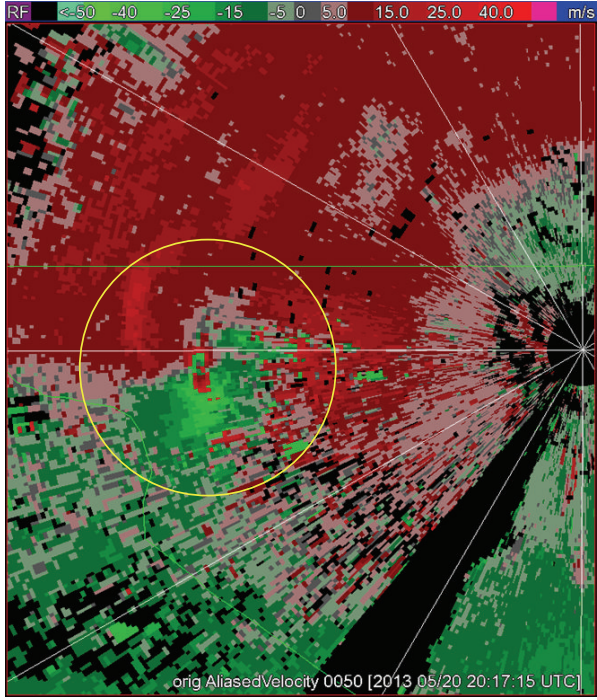

(a)

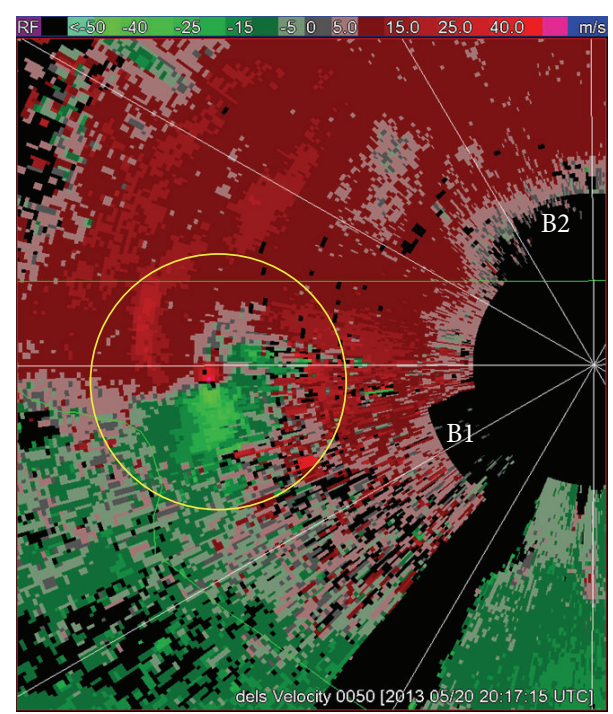

(b)

FiguRE 6: Enlarged displays around the mesocyclone of (a) the raw radial-velocity image in Figure 5(a) and (b) the dealiased radial-velocity image in Figure 5(c). The yellow circle in each panel encircles the mesocyclone. The white letters B1 and B2 in panel (b) mark the two flagged (in black) areas immediately outside the $5 \mathrm{~km}$ range, while the radial velocities within the $5 \mathrm{~km}$ radial range on $0.5^{\circ}$ tilt are not used and thus flagged (in black) to avoid possible ground clutter contaminations.

continuity check in the second step also has a limitation in its dealiased data coverage. In particular, it does not always cover all the data points in every small but critical area of tornadic mesocyclone. This limitation is seen clearly when the raw and dealiased radial-velocity images in Figures 5(a) and 5(c) are enlarged around the mesocyclone in Figures 6(a) and 6(b), respectively. Continued research efforts are being made to reduce the above limitations.

\section{Appendix}

\section{Cyclonic-Rotation Conditions}

(a) $v_{r \max }-v_{r \min }>v_{N}$ and $\varphi_{\max }>\varphi_{\min }$, where $v_{r \text { max }}$ (or $v_{r \text { min }}$ ) is the maximum (or minimum) radial velocity among all the seed data in the $11 \times 41$ window and $\varphi_{\max }$ (or $\left.\varphi_{\min }\right)$ is the azimuthal angle of the $v_{r \max }\left(\right.$ or $v_{r \text { min }}$ ) data point.

(b) $v_{r^{+}}-v_{r^{-}}>0.3 v_{N}$, where $v_{r^{+}}$(or $v_{r^{-}}$) is the averaged value of those seed data that are larger (or smaller) than $v_{r m} \equiv\left(v_{r \max }+v_{r \text { min }}\right) / 2$ in the $11 \times 41$ window.

(c) $\varphi_{+}>\varphi_{-}$, where $\varphi_{+}$(or $\left.\varphi_{-}\right)$is the averaged azimuthal angle for those seed data that are larger (or smaller) than $v_{r m}$ in the $11 \times 41$ window.

(d) $N_{l^{-}}>2 N_{l^{+}}$and $N_{r^{+}}>2 N_{r^{-}}$, where $N_{l^{+}}$(or $N_{l^{-}}$) is the number of seed data that are larger (or smaller) than $v_{r m}$ in the left window, $N_{r^{+}}$(or $N_{r^{-}}$) is the number of seed data that are larger (or smaller) than $v_{r m}$ in the right window, and the $11 \times 41$ window is divided into two parts, the left window and the right window, by the radial line at $\varphi=\varphi_{m} \equiv\left(\varphi_{\max }+\varphi_{\min }\right) / 2$.
If the above four cyclonic-rotation conditions are satisfied for the special datum point being checked, then the $11 \times$ 41 window centered at the special datum point may very likely cover the vortex center of a mesocyclone. In this case, as by-products, the vortex center location can be estimated by $(r, \varphi)=\left(r_{m}, \varphi_{m}\right)$ on the current tilt in the radar local coordinate system, and the radius of maximum tangential velocity for the vortex flow can be estimated by $\rho_{M} \approx$ $\left(\left|r_{\max }-r_{\min }\right|^{2}+r_{m}^{2}\left|\varphi_{\max }-\varphi_{\min }\right|^{2}\right)^{1 / 2} / 2$, where $r_{m} \equiv\left(r_{\max }+\right.$ $\left.r_{\min }\right) / 2$ and $r_{\max }\left(\right.$ or $\left.r_{\min }\right)$ is the radial range of the $v_{r \max }$ (or $\left.v_{r \text { min }}\right)$ data point.

\section{Acknowledgments}

The authors are thankful to Dr. David Stensrud and anonymous reviewers for their comments and suggestions that improved the representation of the results. This research was supported by the NCEP-NSSL radar data QC project and the ONR Grant N000141010778 to the University of Oklahoma (OU). Funding was also provided by DOC/NOAA/OAR under NOAA-OU Cooperative Agreement NA17RJ1227.

\section{References}

[1] M. D. Eilts and S. D. Smith, "Efficient dealiasing of Doppler velocities using local environment constraints," Journal of Atmospheric and Oceanic Technology, vol. 7, no. 1, pp. 118-128, 1990.

[2] G. J. Stumpf, A. Witt, E. D. Mitchell et al., "The national severe storms laboratory mesocyclone detection algorithm for the WSR-88D," Weather and Forecasting, vol. 13, no. 2, pp. 304-326, 1998. 
[3] T. M. Smith and K. L. Elmore, "The use of radial velocity derivative to diagnose rotation and divergence," in Proceedings of the 11th Conference on Aviation, Range, and Aerospace Meterology, pp. 855-861, American Meteorological Society, Hyannis, Mass, USA, October 2004.

[4] Z. Jing and G. Wiener, "Two-dimensional dealiasing of Doppler velocities," Journal of Atmospheric and Oceanic Technology, vol. 10, pp. 798-808, 1993.

[5] D. W. Burgess and T. D. Crum, "Observed failure modes of the WSR-88d velocity dealiasing algorithm during severe weather outbreaks," in Proceedings of the 34rd Conference on Radar Meteorology, American Meteorological Society, Williamsburg, Va, USA, 2009.

[6] A. Witt, R. A. Brown, and Z. Jing, "Performance of a new velocity dealiasing algorithm for the WSR-88D," in Proceedings of the 34rd Conference on Radar Meteorology, American Meteorological Society, Williamsburg, Va, USA, 2009.

[7] J. Gong, L. Wang, and Q. Xu, "A three-step dealiasing method for Doppler velocity data quality control," Journal of Atmospheric and Oceanic Technology, vol. 20, pp. 1738-1748, 2003.

[8] J. Gao, K. K. Droegemeier, J. Gong, and Q. Xu, "A method for retrieving mean horizontal wind profiles from single-Doppler radar observations contaminated by aliasing," Monthly Weather Review, vol. 132, pp. 1399-1409, 2004.

[9] J. Zhang and S. Wang, "An automated 2D multipass Doppler radar velocity dealiasing scheme," Journal of Atmospheric and Oceanic Technology, vol. 23, no. 9, pp. 1239-1248, 2006.

[10] Q. Xu, K. Nai, L. Wei, P. Zhang, S. Liu, and D. Parrish, "A VADbased dealiasing method for radar velocity data quality control," Journal of Atmospheric and Oceanic Technology, vol. 28, no. 1, pp. 50-62, 2011.

[11] Q. Xu and K. Nai, "An adaptive dealiasing method based on variational analysis for radar radial velocities scanned with small Nyquist velocities," Journal of Atmospheric and Oceanic Technology, vol. 29, pp. 1723-1729, 2012.

[12] Q. Xu, K. Nai, and L. Wei, "Fitting VAD winds to aliased doppler radial-velocity observations: a global minimization problem in the presence of multiple local minima," Quarterly Journal of the Royal Meteorological Society, vol. 136, no. 647, pp. 451-461, 2010.

[13] Q. Xu and K. Nai, "A two-step variational method for analyzing severely aliased radar velocity observations with small Nyquist velocities," Quarterly Journal of the Royal Meteorological Society, 2013.

[14] S. Liu, G. DiMego, K. V. Kumar et al., "WSR-88D radar data processing at NCEP," in Proceedings of the 34rd Conference on Radar Meteorology, American Meteorological Society, Williamsburg, Va, USA, 2009.

[15] Q. Xu, K. Nai, L. Wei, and Q. Zhao, "An unconventional approach for assimilating aliased radar radial velocities," Tellus A, vol. 61, no. 5, pp. 621-630, 2009. 

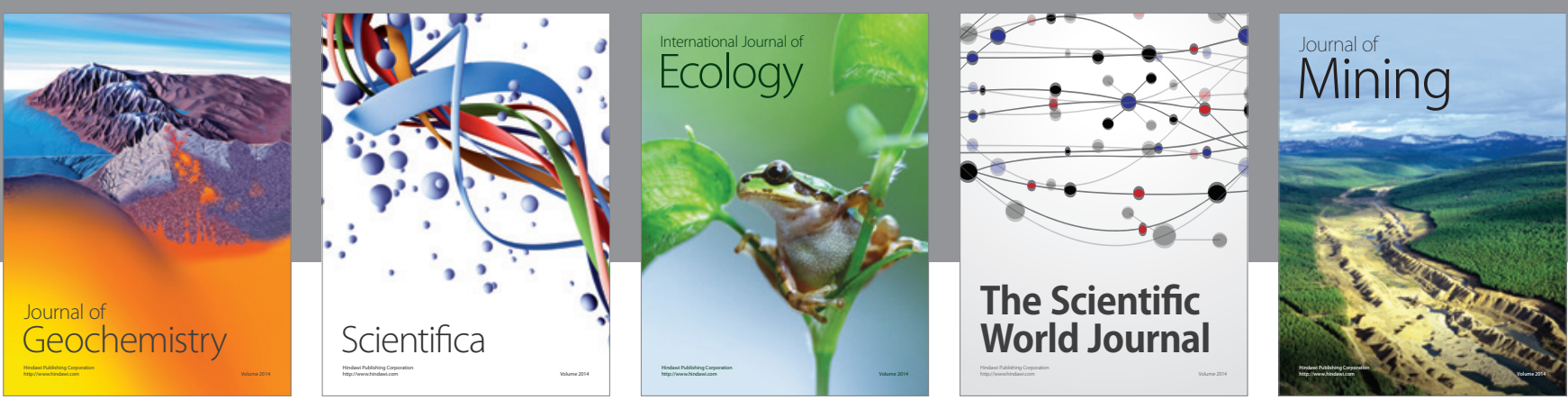

The Scientific World Journal
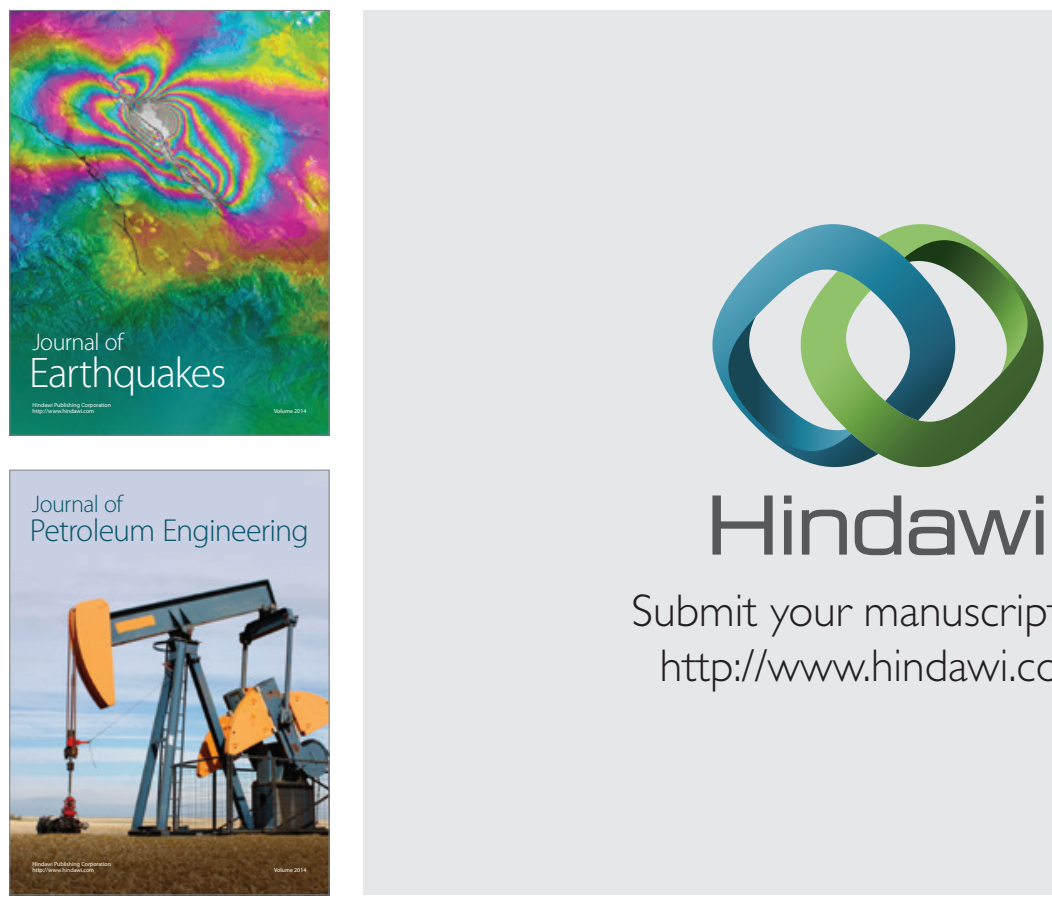

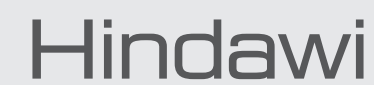

Submit your manuscripts at

http://www.hindawi.com
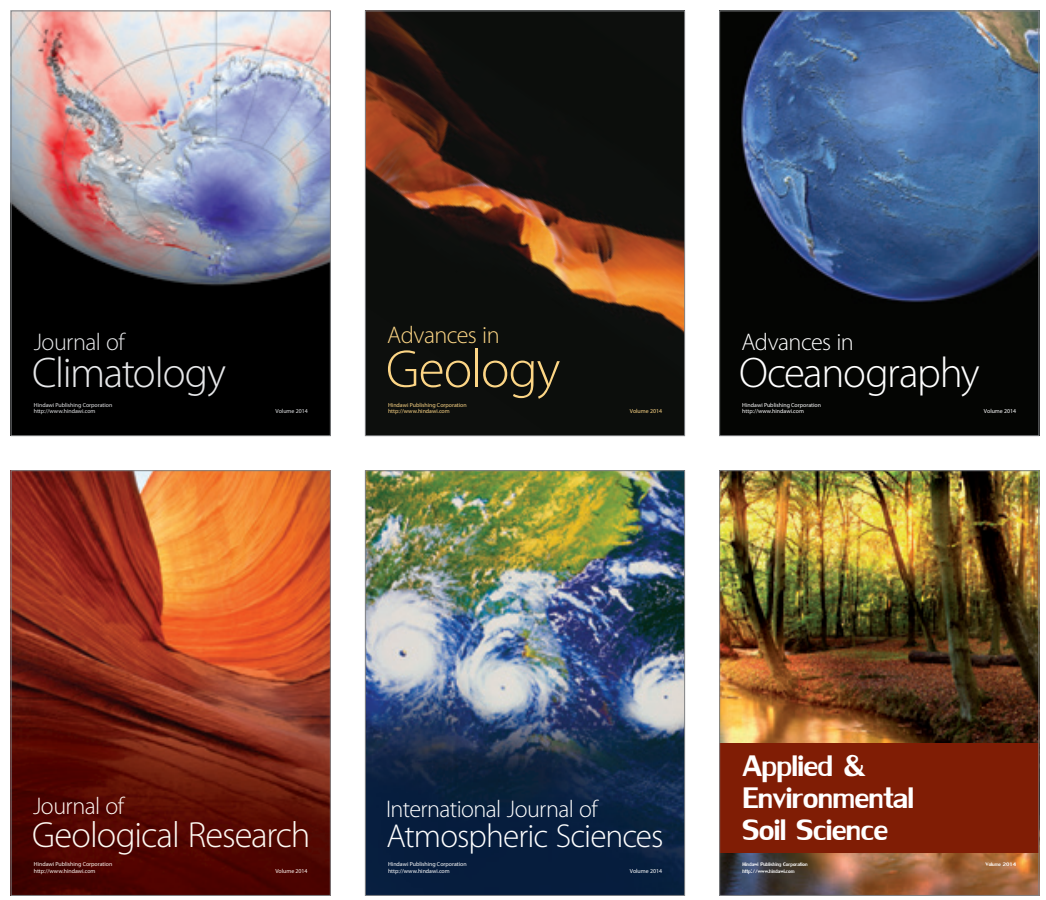
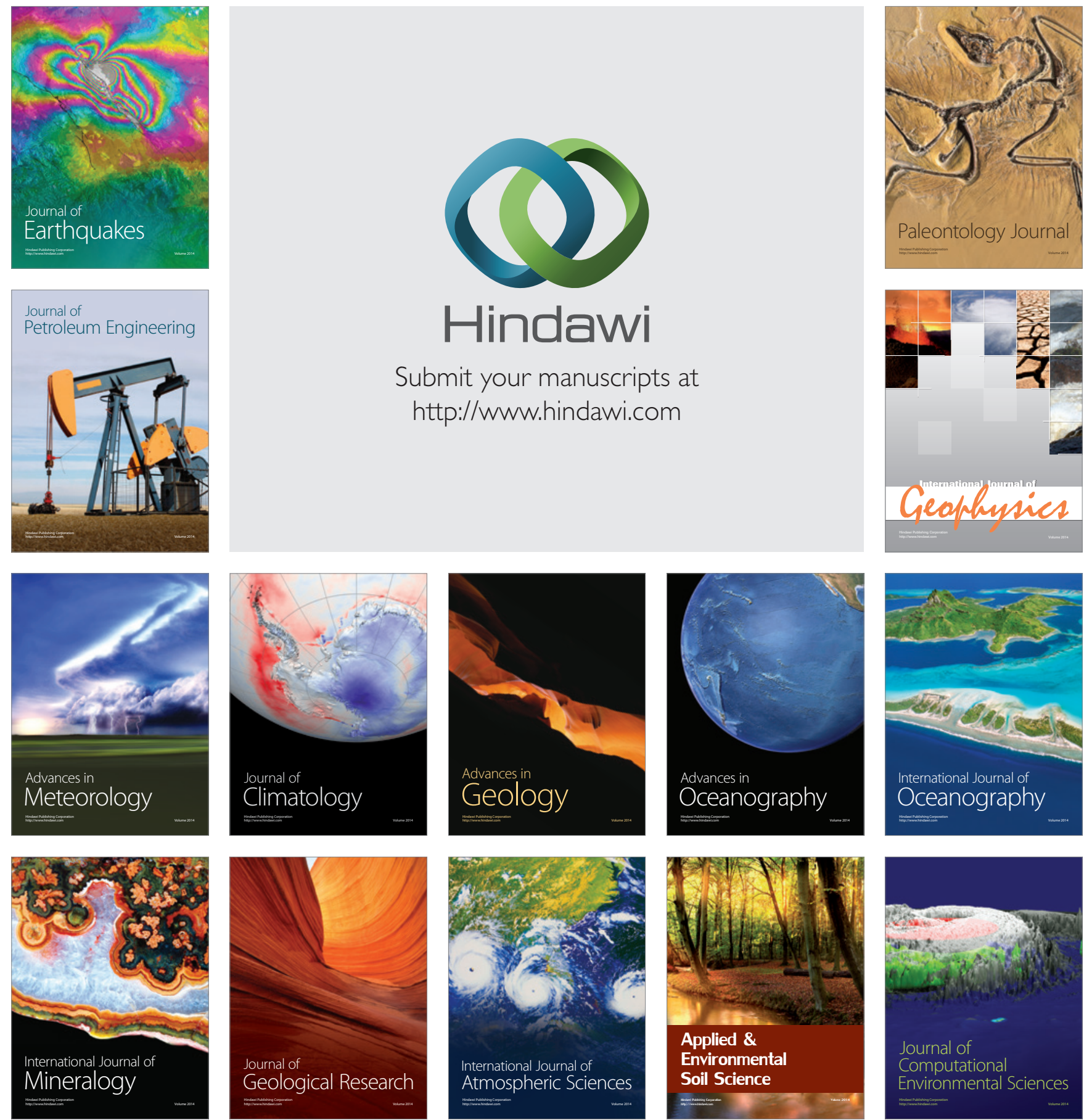